\title{
Chronic electrical stimulation reduces hyperalgesia and associated spinal changes induced by peripheral nerve injury
}

Víctor M. López-Álvarez, PhD; Stefano Cobianchi, PhD; Xavier Navarro, PhD, MD

Institute of Neurosciences, Department of Cell Biology, Physiology and Immunology, Universitat Autònoma de Barcelona, and Centro de Investigación Biomédica en Red sobre Enfermedades Neurodegenerativas (CIBERNED), Bellaterra, Spain.

This work was supported by Grant EPIONE (FP7-602547) from the European Commission (EC), and TERCEL and CIBERNED funds from the Fondo de Investigación Sanitaria of Spain, and FEDER funds.

\section{Authorship and Contribution:}

All authors contributed to the experimental work, writing the paper, and have approved the final version.

\section{Conflict of interest statement}

The authors have no conflicts of interest to declare.

Corresponding author: X. Navarro. Facultat de Medicina, Universitat Autònoma de Barcelona, E-08193 Bellaterra, Spain.E-mail adress: xavier.navarro@uab.cat

This is a non-final version of an article published in final form in Neuromodulation, DOI: 10.1111/NER.12927 


\begin{abstract}
Objectives: we aimed to investigate if different protocols of electrical stimulation following nerve injury might improve neuropathic pain outcomes and modify associated plastic changes at the spinal cord level.

Materials and Methods: Adult rats were subjected to sciatic nerve transection and repair, and distributed in 4 groups: untreated (SNTR, $n=12)$, repeated acute electrical stimulation ( $\mathrm{rAES}, 50 \mathrm{~Hz}, 1 \mathrm{~h}, \mathrm{n}=12$ ), chronic electrical stimulation (CES, $50 \mathrm{~Hz}, 1 \mathrm{~h}, \mathrm{n}=12$ ), and increasingfrequency chronic electrical stimulation (iCES, $1 \mathrm{~h}, \mathrm{n}=12$ ) delivered during 2 weeks following the lesion. The threshold of nociceptive withdrawal to mechanical stimuli was evaluated by means of a Von Frey algesimeter during 3 weeks postlesion. Spinal cord samples were processed by immunohistochemistry for labeling glial cells, adrenergic receptors, $\mathrm{K}^{+}-\mathrm{Cl}^{-}$ cotransporter 2 (KCC2) and GABA.

Results: acute electrical stimulation ( $\mathrm{rAES}, 50 \mathrm{~Hz}, 1 \mathrm{~h}$ ) delivered at 3, 7 and 14 days induced an immediate increase of mechanical pain threshold that disappeared after a few days. Chronic electrical stimulation given daily reduced mechanical hyperalgesia until the end of follow-up, being more sustained with the iCES than with constant $50 \mathrm{~Hz}$ stimulation (CES). Chronic stimulation protocols restored the expression of $\beta 2$ adrenergic receptor and of KCC2 in the dorsal horn, which were significantly reduced by nerve injury. These treatments decreased also the activation of microglia and astrocytes in the dorsal horn.

Conclusion: daily electrical stimulation, especially if frequency-patterned, was effective in ameliorating hyperalgesia after nerve injury, and partially preventing the proinflammatory and hyperalgesic changes in the dorsal horn associated to neuropathic pain.
\end{abstract}

Key words: electrical stimulation, hyperalgesia, neuropathic pain, sciatic nerve, spinal cord 


\section{Introduction}

Neuropathic pain is often resistant to conventional pharmacological therapeutical interventions. Non-pharmacological approaches have been also investigated for their potential in attenuating neuropathic pain. Electrical nerve stimulation, through either percutaneous leads or implantable stimulation devices, has been used as an alternative treatment for a variety of chronic pain conditions. Experimental and clinical studies have provided evidence that electrical stimulation of injured peripheral nerves may be a good option for the control of neuropathic pain, including cases of traumatic nerve damage, neuroma, postherpetic neuralgia and diabetic neuropathy (1-3).

However, the optimal characteristics of electrical stimulation for providing effective analgesia have not been fully defined yet. Peripheral neurostimulation techniques applied differ on the type and location of electrodes and the intensity and frequency of stimulation. These factors, together with the pattern of peripheral nerve activation, may be related to the differences in the therapeutic outcomes reported. The majority of previous studies used low intensity currents to treat neuropathic pain, adjusted to evoke mild non-painful sensations, varying the frequency in a wide range (i.e. 2-100 Hz) (4-7). A constant frequency of $50 \mathrm{~Hz}$ is the most commonly used for pain therapy in clinical protocols. However, an increasing-frequency pattern of sensory inputs induced by physical exercise has been demonstrated to be more effective than a constant rhythm in the reduction of neuropathic pain in animal models (8-9).

The mechanisms of action of neurostimulation remain partially unknown. The original explanation postulated that orthodromic stimulation of large sensory nerve fibers activates interneurons in the dorsal horn of the spinal cord which modulate the processing of nociceptive information, following the gate control theory. Another explanation is that electrical stimulation directly alters the excitability of peripheral nerve fibers, reducing activation of primary nociceptive afferents. It has been also suggested that nerve stimulation modulates neurotransmission at the spinal cord, involving changes in opioid and glutamate receptors (10). In addition, it may influence the activity of descending pain suppressing pathways, acting by releasing catecholamines at the dorsal horn. Indeed, pharmacological stimulation of adrenoceptors (11-12) reduced neuropathic pain after nerve injuries, and $\beta_{2}$ receptors also participate in the reduction of hyperalgesia induced by treadmill running after nerve injury (13). The activation of $\beta_{2}$ receptors may contribute to regulate the activation of 
microglia in the spinal cord induced by nerve injury (14-15), which on turn leads to generation of neuropathic pain. Reactive microglia increase the production of some neurotrophic factors, such as BDNF. BDNF is able to downregulate the $\mathrm{K}^{+}-\mathrm{Cl}^{-}$cotransporter 2 (KCC2) in the dorsal horn neurons, resulting in increased excitability and hyperalgesia (1619). Another mechanism that can be modified by peripheral nerve stimulation is the release of GABA by inhibitory interneurons in the dorsal horn (20). All these possible changes at the dorsal horn network after a nerve injury contribute to the development of neuropathic pain. In this study, we investigated if repeated single sessions or daily and patterned electrical stimulation following nerve injury improve neuropathic pain outcomes and modify some of the potentially involved mechanisms, such as glial reactivity, expression of KCC2 and GABA in the spinal cord.

\section{Materials and methods}

\subsection{Animals and surgery}

Adult female Sprague-Dawley rats $(240 \pm 30 \mathrm{~g})$ were housed in standard cages and kept on standard laboratory food and water ad libitum with a light-dark cycle of $12 \mathrm{~h}$. All experimental procedures were approved by the Ethics Committee of the Universitat Autònoma de Barcelona and followed the guidelines of the European Commission on Animal Care (EU Directive 2010/63/EU). Rats were anesthetized by intraperitoneal (i.p.) injection of ketamine (10 mg/kg, Imalgene 500) and xylazine (1 mg/kg, Rompun), and lidocaine was applied at the skin prior to incision to minimize surgical pain. Rats were submitted to a sciatic nerve transection and repair (SNTR), a well-characterized model that allows the evaluation of neuropathic pain and nerve regeneration (21-22). The right sciatic nerve was exposed at mid-thigh, transected at $92 \mathrm{~mm}$ from the tip of the third toe, and repaired by epineural sutures (10-0 Ethilon), maintaining the fascicular alignment of tibial, peroneal and sural branches.

For delivering electrical stimulation, rats were implanted with a wire system, so they could be stimulated daily without the need of anesthesia. A unipolar system electrode of 1 channel made of stainless steel with ground (MS303/3-AIU/SPC, $5 \mathrm{~cm}$ long, PlasticsOne Inc., Roanoke, VA, USA) was attached to a 3D plastic pedestal. The pedestal was fixed with sutures to the muscle fascia and the skin on the back of the rat (23). The electrode wires were placed in a stress-release loop to allow for leg movements without pulling, and the tips 
were passed under the gluteus maximus muscle to the sciatic nerve. The ground lead was trimmed to a shorter length and the cathode lead was twisted around the sciatic nerve proximal to the lesion. Finally, the wound was closed in layers and disinfected with povidone iodine. Rats were kept in a warm environment until complete recovery from anesthesia.

\subsection{Experimental design}

Animals were randomly divided in 4 groups following SNTR: untreated rats (group SNTR, $n=12$ ), repeated acute electrical stimulation (group $\operatorname{rAES}, n=12$ ), chronic electrical stimulation (group CES, $\mathrm{n}=12$ ), and increasing-frequency chronic electrical stimulation (group iCES, $n=12$ ). A group of naïve rats $(n=6)$ was added for comparison with injured rats.

Electrical stimulation was performed while the rats were free to move in individual standard cages. In the rAES group, stimulation sessions of one hour duration were performed at 3, 7 and 14 days post injury (dpi), delivering pulses of $0.1 \mathrm{~ms}$ duration and suprathreshold intensity ( $\sim \mathrm{V}$ ) at $50 \mathrm{~Hz}$ (Grass S44, Quincy MA). A stimulation frequency of $50 \mathrm{~Hz}$ was chosen based on preliminary studies in which we compared acute stimulation delivered at 2 , 20, 50 and $100 \mathrm{~Hz}$, and found that the $50 \mathrm{~Hz}$ frequency was the most efficient producing an hypoalgesic effect (data not shown). Chronic stimulation treatments (CES and iCES) were delivered one hour daily from 3 until $14 \mathrm{dpi}$. CES was applied following the same pattern of stimulation than rAES. The iCES group had the same timing and duration of stimulation than the CES group, but with a pattern mimicking the locomotion steps performed by rats in an increasing treadmill training protocol previously described $(9,24)$. The frequency of steps was extrapolated and transformed into trains of pulses. Each train was composed of 20 pulses of $0.1 \mathrm{~ms}$ duration and suprathreshold intensity, given at $50 \mathrm{~Hz}$. The train duration was $400 \mathrm{~ms}$ and the train rate was increasing 0.13 trains per second (tps) every 5 minutes from 0.67 tps until 2.1 tps at the end of one hour. The total number of pulses in 1 hour was 99720, and for the full duration of the treatment 1196640 . We observed the slight twitches induced by stimulation on gluteus and biceps femoris muscles, that are close to the stimulation leads, to ensure that stimulation was effectively delivered suprathreshold for A $\alpha \beta$ fibers.

\subsection{Assessment of sensory function}


Three days before surgery, all the injured animals were habituated to the experimental devices, and then tested for baseline values. The threshold of nociceptive responses to mechanical stimuli was evaluated on both hindpaws by means of algesimetry tests at $0,3,7$, 14 and $21 \mathrm{dpi}$. Algesimetry tests were performed before, immediately after (-post), one hour $(-$ post $+1 h)$ and five hours (-post+5h) after treatment sessions (rAES, CES and iCES) to evaluate the evolution of the hypoalgesic effects along a short period of time following the stimulation session.

Two different areas of the plantar surface of the hindpaw were tested: the lateral (innervated by tibial and sural nerves) and the medial (innervated by tibial and saphenous nerves) regions (22). Sensibility to mechanical stimuli was measured by means of an electronic Von Frey algesimeter (Bioseb, Chaville, France). Rats were placed on a wire net platform in plastic chambers $30 \mathrm{~min}$ before testing for habituation. Then, a non-noxious pointed probe was gently applied to each test site, slowly increasing the pressure. The threshold was expressed as the force (in grams) at which rats withdrew the paw in response to the stimulus. A cutoff force was set at $45 \mathrm{~g}$ when the stimulus lifted the paw without response. The mechanical nociceptive threshold was calculated as the mean of three measurements per paw region, obtained with 3 min intervals, and then used for calculating the percentage of the threshold of injured vs. contralateral intact hindpaw at each test day. This allows good comparison of hyperalgesia induced by the injury, since no significant variations of the contralateral threshold were found in previous studies $(9,22,24)$.

\subsection{Immunohistochemistry of spinal cord}

At the end of the treatment at $14 \mathrm{dpi}$ (half of the rats per group) and at $21 \mathrm{dpi}$ (for the rest, to see possible variations one week after cessation of the treatment), animals were euthanized, and spinal cord samples were collected for immunohistochemical assays. Lumbar L4-L5 spinal cord segments were removed from paraformaldehyde 4\% perfused animals, kept in fixative for 1 hour and then cryoprotected with PBS containing 30\% sucrose. Transverse sections were cut at $25 \mu \mathrm{m}$ thickness on a cryostat and mounted on slides. Sections were blocked with specific serum and incubated overnight with primary antibodies in $0.3 \%$ Triton $X-100$ in PBS, for staining microglial cells (anti-lba1, goat, 1:200, Abcam), astrocytes (anti-GFAP, rabbit, 1:500, DakoCytomation), adrenergic receptors (anti- $\beta_{2}$ receptors, rabbit, 1:500, Santa Cruz), $\mathrm{K}^{+}-\mathrm{Cl}^{-}$cotransporter 2 (anti-KCC2, rabbit, 1:500, 
Millipore) and $\gamma$-aminobutyric acid (anti-GABA, rabbit, 1:500, Sigma-Aldrich). The following day, sections were incubated for 2 hours with Alexa Fluor 488 and/or Alexa Fluor 594 conjugated secondary antibodies (1:200, Life Technologies). After washing in PBS, sections were coverslipped with a solution of DAPI in Mowiol mounting medium.

For each marker, three sections from 4 samples per group and time point were used for quantifying immunolabeling. 20X images were captured with a Zeiss LSM 700 confocal microscope, and analyzed using ImageJ software (NIH, USA). Thresholding of fluorescent signal was adjusted over the background level of a negative control. The integrated density of immunoreactivity was calculated within regions of interest drawn with ImageJ software tool. Data are shown for both ipsilateral and contralateral sides.

\subsection{Data analysis}

Data are presented as mean \pm SEM. Statistical analysis of nociceptive thresholds was made by two-way analysis of variance (ANOVA) with group and time after injury as factors, followed by Bonferroni's post hoc comparisons. Statistical comparisons for immunofluorescence data were made by two-way ANOVA followed by Tukey's post hoc test. The level of statistical significance was $5 \%(p<0.05)$.

\section{Results}

\subsection{Hyperalgesia is reduced by chronic electrical stimulation after SNTR}

Changes in sensory thresholds were recorded at both medial and lateral sites of the hindpaw to monitor the contribution to hyperalgesia of collateral sprouting of the intact saphenous nerve, and of denervation by the injured sciatic nerve. After SNTR the lateral side of the injured hindpaw was unresponsive during the 21 days follow-up, since this territory remains denervated until at least 4 weeks after injury (data not shown) $(22,24)$. On the contrary, sciatic nerve injury produced a marked decrease of the mechanical pain threshold at the medial side of the hindpaw from 3 to $21 \mathrm{dpi}$ (Fig. 1A-C).

Repeated-acute electrical stimulation, consisting in 1 hour stimulation given at 3, 7 and 14 dpi, induced a rapid but temporary hypoalgesic effect in the rAES group. Immediately after stimulation, the reduction of the mechanical threshold was significantly prevented $(p<0.05)$, but this effect declined after 5 hours and was completely lost at the pre-stimulation tests 
performed at 7 and $14 \mathrm{dpi}$ (Fig. 1A). On the other hand, applying chronic daily treatments by CES (Fig. 1B) and iCES (Fig. 1C), the prevention of hyperalgesia was more sustained along the time. The maintenance of the immediate effect over time poststimulation was enhanced in the iCES group slightly more than in the CES group, and in both significantly more than in the rAES group.

\subsection{Chronic electrical stimulation reduced glial cell reactivity in the dorsal horn after SNTR}

After SNTR, there was an increase in immunoreactivity for GFAP, showing hypertrophied astrocytes in the dorsal horn of the spinal cord in both sides compared to naïve rats (Fig. $2 A, B)$. Repeated-acute electrical stimulation had no effect in reducing this increase of astrocyte reactivity at 14 or $21 \mathrm{dpi}$ (Fig. 2B). However, both chronic electrical stimulation treatments induced a clear reduction in GFAP expression to levels similar to naïve animals. After SNTR, there was also marked microglial reactivity, evidenced by an increase in Iba1 immunoreactivity in the dorsal horn compared to naïve rats (Fig. 3A). rAES treatment showed no significant differences with respect to the SNTR group at 14 or $21 \mathrm{dpi}$ (Fig. 3B). iCES treatment counteracted the increase of Iba1 expression in the ipsilateral dorsal horn at 14 and 21 dpi (Fig. 3B; p<0.05), and CES treatment only at 21 dpi (Fig. 3B; $p<0.05$ ), compared to SNTR rats. There was a tendency, although not significant, to reduce Iba1 overexpression with chronic stimulation treatments in the contralateral dorsal horn.

\subsection{Chronic electrical stimulation reverted the decrease of $K C C 2$ expression in the dorsal horn} after SNTR

The decline in KCC2 expression following pathological changes in glial cells activity results in neuronal hyperexcitability due to a decline of the normally inhibitory interneurons (16). After SNTR we found a marked and sustained reduction of KCC2 levels in the dorsal horn bilaterally for at least 3 weeks (Fig. 4A), in agreement with a previous report (19). Chronic but not acute repeated electrical stimulation protocols were effective reverting the decrease of KCC2. CES and iCES significantly counteracted the KCC2 reduction in the injured side at 14 and $21 \mathrm{dpi}$, and iCES also in the contralateral side at $21 \mathrm{dpi} \mathrm{compared} \mathrm{to} \mathrm{untreated} \mathrm{SNTR} \mathrm{rats}$ (Fig. 4B; $p<0.05$ ). 
3.4. Chronic electrical stimulation reverted the decrease of $\beta_{2}$ receptor expression in the dorsal horn after SNTR

We found the $\beta_{2}$ receptor expressed in laminae I-III of the dorsal horn in the naïve rats (Fig. 5A). SNTR reduced the expression of $\beta_{2}$ receptor in both the ipsilateral and contralateral dorsal horn laminae. The expression of $\beta_{2}$ was not restored by rAES but chronic stimulation with CES and iCES protocols was effective in maintaining $\beta_{2}$ expression levels close to naïve values in the ipsilateral side at 14 and $21 \mathrm{dpi}$ (Fig 5B; $\mathrm{p}<0.05$ ). In the contralateral side, only iCES treatment counteracted the decrease of $\beta_{2}$ expression levels after SNTR (Fig. 5B; $\mathrm{p}<0.05)$.

\subsection{Chronic electrical stimulation prevented the decrease of GABA in dorsal horn after SNTR} Because of the relation between adrenergic receptors and GABA in spinal inhibition (25) and a previous report showing that GABA release in the spinal cord mediated the hypoalgesic effect of transcutaneous electrical nerve stimulation (TENS) (Maeda et al., 2007), we studied the expression of GABA in the spinal cord dorsal horn. We found that the expression of GABA in lamina I, II and III was reduced in both sides of the spinal cord at 14 and 21 days after SNTR. CES and iCES, but not rAES, treatments counteracted the decrease of GABA in the dorsal horn after SNTR in both sides at 14 and 21 dpi (Fig. 6B; $p<0.05$ ).

\section{Discussion}

The results of this study demonstrate the beneficial effects of three different patterns of electrical stimulation delivered to the injured sciatic nerve on the signs of hyperalgesia and associated changes in the nociceptive pathway. Interestingly, the hypoalgesic effects were persistent only when stimulation was applied daily during at least 2 weeks after injury (CES and iCES) but not when stimulation sessions were repeated at intervals of several days (rAES).

\subsection{Effects of chronic electrical stimulation on neuropathic pain}

Peripheral nerve stimulation has been reported as an effective treatment for individuals with a variety of pain conditions, particularly for patients with neuropathic pain (1-2, 26-27). Previous studies showed that, irrespective of the methodology used (in terms of frequency, intensity and duration of application), peripheral electrical stimulation inhibited hyperalgesia 
in rat models of peripheral neuropathy $(10,28-30)$. The frequency of stimulation may be a key factor. Although there are different studies of nerve stimulation for neuropathic pain showing good results in reducing hyperalgesia by applying different frequencies $(5-7,31)$, we focused in $50 \mathrm{~Hz}$ because is the most commonly used for pain therapies in the clinic and has been validated in preclinical models of neuropathic pain (see 32,33 ) and supported by our preliminary assays. Alternating the frequencies of stimulation induces a variation in the pain thresholds (34) and high frequencies are known to be able to block conduction in C-fibers (35). Activity dependent treatments, like treadmill running, have been also demonstrated to be effective in reducing neuropathic pain and one of the most efficient treadmill protocols for injured rats consists in an increasing-intensity (velocity) exercise (iTR) (8-9, 24). For both types of activity-dpeendent therapies, an early start is important, since this may prevent the development of plastic changes in spinal circuitry and neuroinflammatory responses induced by the nerve injury $(8,9,36)$. Taking these works into account, we mimicked the inputs of the running locomotion of the rats and we extrapolated the iTR to a protocol of stimulation named iCES, in which the rats are stimulated with trains of pulses increasing in frequency over one hour per session. The results we found in this study present this chronic pattern as the most effective in reducing mechanical hyperalgesia after sciatic nerve injury compared to rAES and CES treatments. The effect of one session of peripheral electrical stimulation was shown previously to last up to 12 hours, and repetitive stimulation required for a cumulative effect (10), in agreement with our results. Interestingly, in our protocols, iCES and to a lesser degree CES produced long-lasting hypoalgesia at 21 days despite the treatment was interrupted at 14 days after the lesion.

\subsection{Effects of chronic electrical stimulation on glial reactivity in the dorsal horn after SNTR}

Peripheral nerve injuries provoke changes in spinal microglia including proliferation, hypertrophy, and overexpression of cytokines and chemokines, that contribute to sustained plastic changes in the dorsal horn favoring pain sensation $(37,38)$. Microglia play a role in the initiation phase of neuropathic pain, and their proliferation is related with the development of neuropathic pain (39). Activation of microglia has been described to be regulated by $\beta_{2}$ adrenergic receptor activation (14-15) and plays an active role in the pathway that leads to BDNF overproduction and KCC2 dephosphorylation in neuropathic pain states (19). We evaluated microglial activation after SNTR and we found a significant 
capacity of chronic stimulation treatments to reduce microglial activation in the dorsal horn of the spinal cord after injury. In this regard, activation of astrocytes is also involved in neuropathic pain, especially during the chronic phase of pain. Our data showed that suppression of astrocyte activation in the spinal cord after SNTR also occurred only by chronic but not acute electrical stimulation treatment.

\subsection{Effects of chronic electrical stimulation on the expression of biomarkers in the dorsal horn after SNTR}

Activated microglia is known to release BDNF after a nerve injury that triggers neuropathic mechanisms, such as downregulation of $\operatorname{KCC} 2(16,17,19)$ resulting in neuronal excitation when normally inhibitory GABAergic synapses are activated after peripheral nerve injury. Activation of GABAergic inputs to neurons in which the KCC2 transporter is downregulated results in markedly increased levels of spontaneous activity $(40,41)$. Our work supports previous studies with other activity-dependent treatments, such as iTR (9), that prevented the decreased expression of KCC2 after nerve injury.

On the other hand, adrenergic neurotransmission is implicated in the hypoalgesic effects of antidepressants as well as of electrical spinal cord stimulation (SCS) $(42,43)$. Electrical stimulation induces release of noradrenaline in the spinal cord that acts on adrenergic receptors; $\alpha 1$ and $\alpha 2$ adrenoceptors have been shown to be involved in pain modulation and in neuropathic pain (44), but the $\beta_{2}$ receptor is also expressed in the dorsal horn of the spinal cord (45). Moreover, pharmacological stimulation of $\beta_{2}$ receptor suppressed neuropathic pain after sciatic nerve insult (12). In a previous study, we found that iTR training strongly increased the expression of $\beta_{2}$ receptor after peripheral nerve injury, participating in the iTRinduced reduction of hyperalgesia (13). The present results also indicate that chronic electrical stimulation protocols are able to counteract the reduction of $\beta_{2}$ receptor expression after SNTR. Activation of $\beta_{2}$ receptor plays a role in the modulation of the microglial inflammatory response to nerve injury (13).

4.4. Effects of chronic electrical stimulation on the expression of GABA in the dorsal horn after SNTR

Alterations in dorsal horn network connectivity provoked by a progressive loss of inhibitory mechanisms contribute to the progression of neuropathic pain. Following a peripheral nerve 
lesion there is loss of A-fiber mediated inhibition on nociceptive dorsal horn neurons (46), that can be explained in part by the reduction in GABA-mediated inhibitory postsynaptic currents in the dorsal horn due to the death of GABAergic interneurons and reduction in the amount of GABA released into the dorsal horn (47).

In this sense, it was reported that low frequency TENS induces release of serotonin in the spinal cord resulting in increased release of GABA that subsequently decreases activity of dorsal horn neurons. Also, high frequency TENS activates supraspinal $\delta$ opioid receptors that release GABA and enkephalins in the spinal cord resulting in decreased release of glutamate and decreased activity of dorsal horn neurons (20). Other works reported that noradrenaline enhances the spinal GABAergic and cholinergic transmission by activating $\alpha 1$ and $\alpha 2$ adrenoreceptors (48). Our data suggest that chronic electrical stimulation triggers a series of changes in the spinal cord such as the restoration of expression of KCC2 and $\beta_{2}$ receptor that act facilitating the increase of GABA release in the spinal cord and the reduction of pain transmission after SNTR.

\section{Conclusions}

The results of this study bring new knowledge on the contribution of peripheral nerve electrical stimulation in reducing neuropathic pain. Through restoration of $\beta_{2}$ receptor expression and reduction of activated glial cells, daily stimulation during 2 weeks after nerve injury seems to act preventing the KCC2 down-regulation, leading to a higher release of GABA and reduction of the hyperalgesic state. Future studies are needed to understand more about these interactions and which neuronal populations and molecular mechanisms are involved. Patterned stimulation as with our increasing-frequency chronic electrical stimulation protocol shows promise towards improving the effectiveness of electrical stimulation therapy in clinical applications.

Abbreviations: SNTR, sciatic nerve transection and repair; rAES, repeated single electrical stimulation; CES, chronic electrical stimulation; iCES, increasing-frequency chronic electrical stimulation; $\mathrm{KCC} 2, \mathrm{~K}^{+}-\mathrm{Cl}^{-}$cotransporter 2; GABA, $\mathrm{\gamma}$-aminobutyric acid; BDNF, brain-derived neurotrophic factor; GFAP, Glial fibrillary acidic protein; i.p., intraperitoneal; dpi, days post injury; TENS, transcutaneous electrical stimulation; iTR, increasing-intensity treadmill exercise; SCS, spinal cord stimulation. 


\section{Acknowledgements}

This work was supported by Grant EPIONE (FP7-602547) from the European Commission (EC), and TERCEL and CIBERNED funds from the Fondo de Investigación Sanitaria of Spain, and FEDER funds. The authors are grateful to Monica Espejo for lab management and the technical help of Nuria Barba for the confocal microscopy. 


\section{References}

1. Slavin KV. Peripheral nerve stimulation for neuropathic pain. Neurotherapeutics 2008;5:100-106.

2. Eisenberg $E$, Waisbrod H, Gerbershagen $\mathrm{HU}$, Long-term peripheral nerve stimulation for painful nerve injuries. Clin J Pain 2004;20:143-146.

3. Novak CB, Mackinnon SE. Outcome following implantation of a peripheral nerve stimulator in patients with chronic nerve pain. Plast Reconstruct Surg 2000;105:19671972.

4. Dabby R, Sadeh M, Goldberg I, Finkelshtein, V. Electrical stimulation of the posterior tibial nerve reduces neuropathic pain in patients with polyneuropathy. J Pain Res 2017;10:2717-2723.

5. Maeda Y, Wacnik PW, Sluka KA. Low frequencies, but not high frequencies of bi-polar spinal cord stimulation reduce cutaneous and muscle hyperalgesia induced by nerve injury. Pain 2008;138:143-152.

6. Jutzeler CR, Curt A, Kramer JLK. Effectiveness of high-frequency electrical stimulation following sensitization with capsaicin. J Pain 2015;16:595-605.

7. Sandkühler J, Chen JG, Cheng G, Randić M. Low-frequency stimulation of afferent Adelta-fibers induces long-term depression at primary afferent synapses with substantia gelatinosa neurons in the rat. J Neurosci 1997;17:6483-6491.

8. Cobianchi S, Marinelli S, Florenzano, Pavome F, Luvisetto S. Short- but not long-lasting treadmill running reduces allodynia and improves functional recovery after peripheral nerve injury. Neuroscience 2010;168:273-287.

9. López-Álvarez VM, Mòdol L, Navarro X, Cobianchi S. Early increasing-intensity treadmill exercise reduces neuropathic pain by preventing nociceptor collateral sprouting and disruption of chloride cotransporters homeostasis after peripheral nerve injury. Pain 2015;156:1812-1825.

10. Sun RQ, Wang HC, Wan Y, Jing Z, Luo F, Han JS et al. Suppression of neuropathic pain by peripheral electrical stimulation in rats: A-opioid receptor and NMDA receptor implicated. Exp Neurol 2004;187:23-29.

11. Stone LS, Vulchanova L, Riedl MS, Wang J, Williams FG, Wilcox GL et al. Effects of peripheral nerve injury on alpha-2A and alpha-2C adrenergic receptor immunoreactivity in the rat spinal cord. Neuroscience 2009;93:1399-1407.

12. Yalcin I, Tessier LH, Petit-Demoulière N, Waltisperger E, Hein L, Freund-Mercier MJ et al. Chronic treatment with agonists of $\beta 2$-adrenergic receptors in neuropathic pain. Exp Neurol 2010;221:115-121.

13. López-Alvarez VM, Puigdomenech $M$, Navarro X, Cobianchi S. Monoaminergic descending pathways contribute to modulation of neuropathic pain by increasingintensity treadmill exercise after peripheral nerve injury. Exp Neurol 2018;299:42-55.

14. Fujita H, Tanaka J, Maeda N, Sakanaka M. Adrenergic agonists suppress the proliferation of microglia through b2-adrenergic receptor. Neurosci Lett 1998;242:37-40.

15. O’Donnell J, Zeppenfeld D, McConnell E, Pena S, Nedergaard M. Norepinephrine: A 
neuromodulator that boosts the function of multiple cell types to optimize CNS performance. Neurochem Res 2012;37:2496-2512.

16. Coull JAM, Beggs S, Boudreau D, Boivin D, Tsuda M, Inoue $K$ et al. BDNF from microglia causes the shift in neuronal anion gradient underlying neuropathic pain. Nature 2015;438:1017-1021.

17. Ferrini F, De Koninck Y. Microglia control neuronal network excitability via BDNF signaling. Neural Plast 2013;2013:429815.

18. Lu Y, Zheng J, Xiong L, Zimmermann M, Yang J. Spinal cord injury-induced attenuation of GABAergic inhibition in spinal dorsal horn circuits is associated with down-regulation of the chloride transporter KCC2 in rat. J Physiol 2008;58623:5701-5715.

19. Mòdol L, Cobianchi S, Navarro X. Prevention of NKCC1 phosphorylation avoids downregulation of $\mathrm{KCC} 2$ in central sensory pathways and reduces neuropathic pain after peripheral nerve injury. Pain 2014;155:1577-1590.

20. Maeda Y, Lisi TL, Vance CGT, Sluka KA. Release of GABA and activation of GABAA in the spinal cord mediates the effects of TENS in rats. Brain Res 2007;1136:43-50.

21. Casals-Díaz L, Vivó M, Navarro X. Nociceptive responses and spinal plastic changes of afferent C-fibers in three neuropathic pain models induced by sciatic nerve injury in the rat. Exp Neurol 2009;217:84-95.

22. Cobianchi S, de Cruz J, Navarro X. Assessment of sensory thresholds and nociceptive fiber growth after sciatic nerve injury reveal the differential contribution of collateral reinnervation and nerve regeneration to neuropathic pain. Exp Neurol 2014;255:1-11.

23. Wurth S, Capogrosso M, Raspopovic S, Gandar J, Federici G, Kinany N et al. Long-term usability and bio-integration of polyimide-based intra-neural stimulating electrodes. Biomaterials 2017;122:114-129.

24. Cobianchi S, Casals-Diaz L, Jaramillo J, Navarro X. Differential effects of activity dependent treatments on axonal regeneration and neuropathic pain after peripheral nerve injury. Exp Neurol 2013;240:157-167.

25. Gassner M, Ruscheweyh R, Sandkühler J. Direct excitation of spinal GABAergic interneurons by noradrenaline. Pain 2009;145:204-210.

26. Verrills $P$, Russo M. Peripheral nerve stimulation for back pain. Prog Neurol Surg 2015;29:127-138.

27. Johansson F, Almay BG, Von Knorring L. Predictors for the outcome of treatment with high frequency transcutaneous electrical nerve stimulation in patients with chronic pain. Pain 1980;9:55-61.

28. Inoue T, Takenoshita M, Shibata M, Nishimura M, Sakaue G, Shibata SC et al. Longlasting effectt of transcutaneous electrical nerve stimulation on the thermal hyperalgesia in the rat model of peripheral neuropathy. J Neurol Sci 2003;211:43-47.

29. Somers DL, Clemente FR. Contralateral high or a combination of high- and lowfrequency transcutaneous electrical nerve stimulation reduces mechanical allodynia and alters dorsal horn neurotransmitter content in neuropathic rats. J Pain 2009;10:221-229.

30. Nam TS, Choi Y, Yeon DS, Leem JW, Paik KS. Differential antinociceptive effect of 
transcutaneous electrical stimulation on pain behavior sensitive or insensitive to phentolamine in neuropathic rats. Neurosci Lett 2001;301:17-20.

31. Bergeron-Vézina K, Corriveau H, Martel M, Harvey MP, Léonard G. High- and lowfrequency transcutaneous electrical nerve stimulation does not reduce experimental pain in elderly individuals. Pain 2015;156:2093-2099.

32. Smits $\mathrm{H}$, van KM, Holsheimer J, Joosten EA. Experimental spinal cord stimulation and neuropathic pain: mechanism of action, technical aspects, and effectiveness. Pain Pract 2013; 13:154-168.

33. Sdrulla $A D, X u Q$, He SQ, Tiwari V, Yang F, Zhang C, et al. Electrical stimulation of lowthreshold afferent fibers induces a prolonged synaptic depression in lamina II dorsal horn neurons to high-threshold afferent inputs in mice. Pain 2015;156:1008-1017.

34. Ward AR, Robertson VJ. Sensory, motor, and pain thresholds for stimulation with medium frequency alternating current. Arch Phys Med Rehabil 1998;79:273-278.

35. Joseph L, Butera RJ. High-frequency stimulation selectively blocks different types of fibers in frog sciatic nerve. IEEE Trans Neural Syst Rehabil Eng 2001;19:550-557.

36. Matsuo H, Uchida K, Nakajima H, Guerrero AR, Watanabe S, Takeura N, et al. Early transcutaneous electrical nerve stimulation reduces hyperalgesia and decreases activation of spinal glial cells in mice with neuropathic pain. Pain 2014;155:1888-901.

37. Calvo M, Dawes JM, Bennett DLH. The role of the immune system in the generation of neuropathic pain. Lancet Neurol 2012;11:629-642.

38. Inoue K, Tsuda M. Microglia and neuropathic pain. Glia 2009;57:1469-1479.

39. Echeverry S, Shi XQ, Zhang J. Characterization of cell proliferation in rat spinal cord following peripheral nerve injury and the relationship with neuropathic pain. Pain 2008;135:37-47.

40. Keller AF, Beggs S, Salter MW, De Koninck Y. Transformation of the output of spinal lamina I neurons after nerve injury and microglia stimulation underlying neuropathic pain. Mol Pain 2007;3:27.

41. Lavertu G, Côté SL, De Koninck Y. Enhancing K-Cl co-transport restores normal spinothalamic sensory coding in a neuropathic pain model. Brain 2014;137:724-738.

42. Nakajima K, Obata H, Iriuchijima N, Saito S. An increase in spinal cord noradrenaline is a major contributor to the antihyperalgesic effect of antidepressants after peripheral nerve injury in the rat. Pain 2012;153:990-997.

43. Schechtmann G, Song Z, Ultenius, Meyerson BA, Linderoth B. Cholinergic mechanisms involved in the pain relieving effect of spinal cord stimulation in a model of neuropathy. Pain 2008;139:136-145.

44. Millan MJ. Descending control of pain. Prog Neurobiol 2002;66:355-474.

45. Nicholson R, Dixon AK, Spanswick D, Lee K. Noradrenergic receptor mRNA expression in adult rat superficial dorsal horn and dorsal root ganglion neurons. Neurosci Lett 2005;380:316-321.

46. Woolf CJ, Wall PD. Chronic peripheral nerve section diminishes the primary afferent Afibre mediated inhibition of rat dorsal horn neurons. Brain Res 1982;242:77-85. 
47. von Hehn CA, Baron R, Woolf CJ. Deconstructing the neuropathic pain phenotype to reveal neural mechanisms. Neuron 2012;73:638-652.

48. Baba H, Shimoji K, Yoshimura M. Norepinephrine facilitates inhibitory transmission in substantia gelatinosa of adult rat spinal cord (part 1): effects on axon terminals of GABAergic and glycinergic neurons. Anesthesiology 2000;92:473-484. 


\section{Figures}
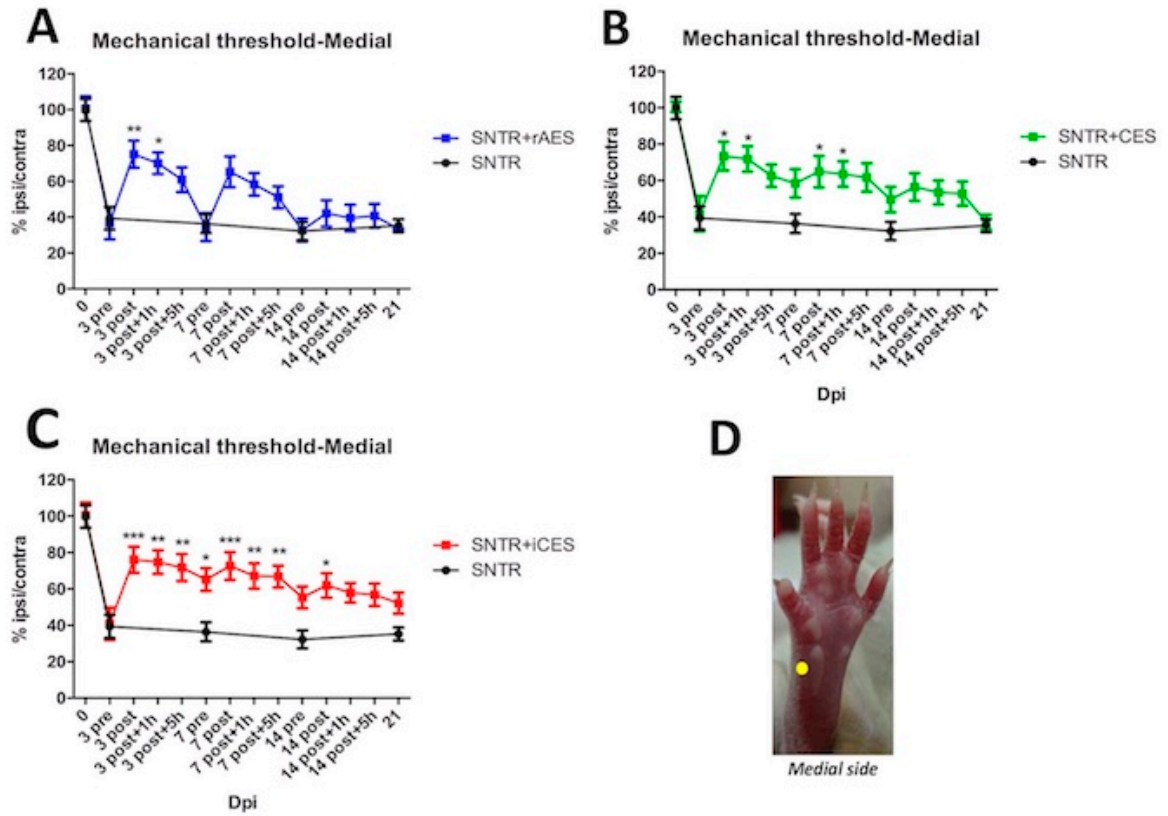

Figure 1. Hyperalgesia is reduced by chronic stimulation after SNTR. Changes in mechanical sensory thresholds recorded at the medial (D) test site in rAES (A), CES (B) and iCES groups (C) after SNTR from $3 \mathrm{dpi}$ pre-stimulation to $21 \mathrm{dpi}$. Recordings were performed before and also post-stimulation and at 1 and 5 hours post-stimulation. Values are represented as the percent ratio between the mean ipsilateral and the contralateral paw. ${ }^{*} p<0.05,{ }^{* *} p<0.01$, $* * * p<0.001$.
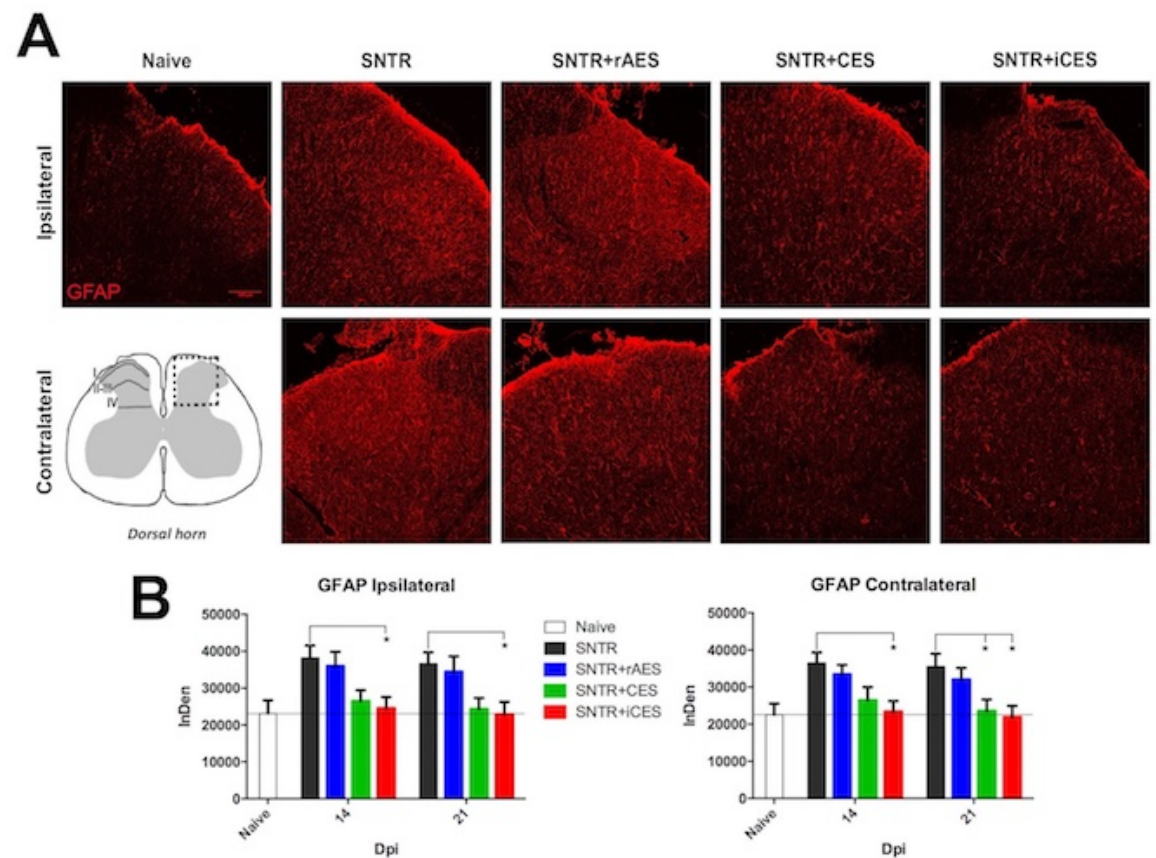

Figure 2. Chronic stimulation reduced GFAP overexpression in dorsal horn after SNTR. (A) Representative confocal images of GFAP immunoreactivity in the spinal cord dorsal horn of naïve rats, and in the ipsilateral and contralateral dorsal horns at $14 \mathrm{dpi}$ of SNTR, SNTR+rAES, SNTR+CES and SNTR+iCES rats, with graphic representation of laminae I, II and III as regions 
of interest considered for quantification. Scale bar $100 \mu \mathrm{m}$. (B) Quantification of GFAP immunoreactivity in the ipsilateral and contralateral dorsal horn of naïve, and SNTR rats compared with the electrical stimulation treatments at 14 and $21 \mathrm{dpi}$. ${ }^{*} \mathrm{p}<0.05$.

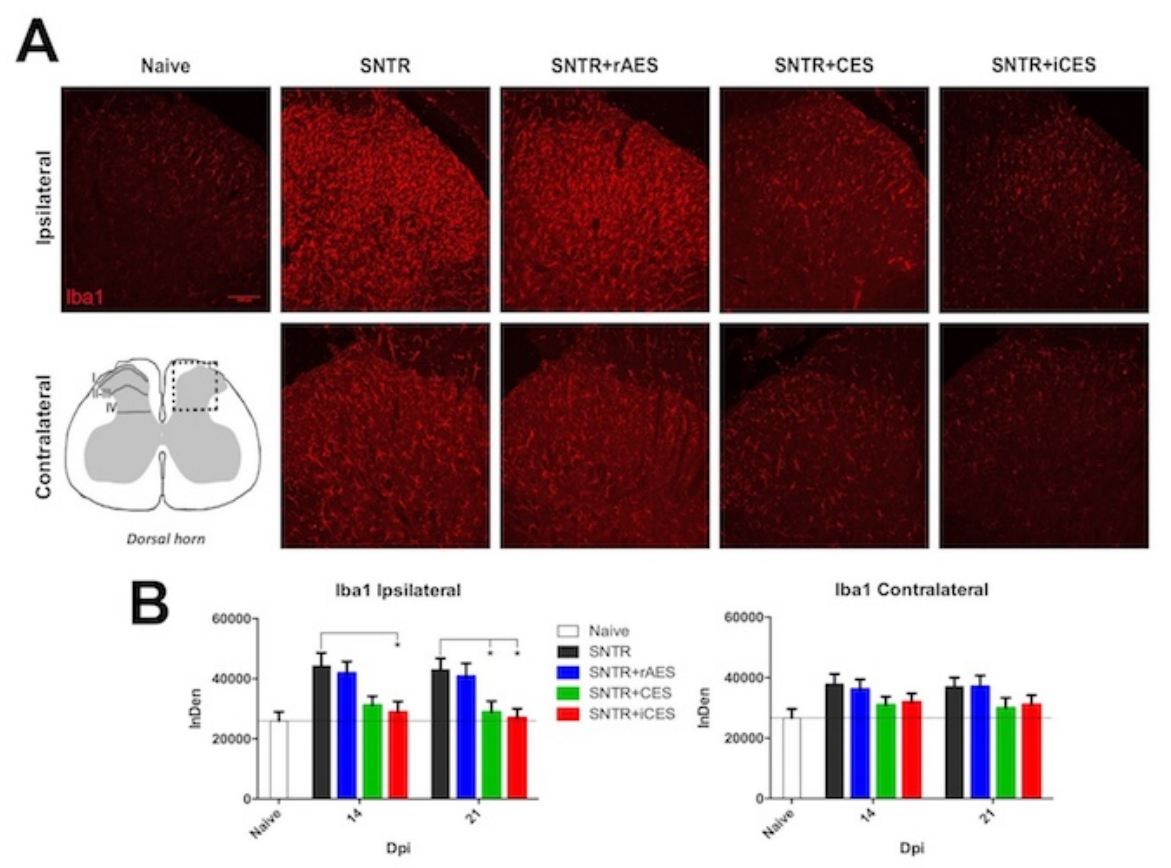

Figure 3. Chronic stimulation counteracted the increase of Iba1 expression in the ipsilateral dorsal horn after SNTR. (A) Representative confocal images of Iba1 immunoreactivity in the spinal cord dorsal horn of naïve rats, and in the ipsilateral and contralateral dorsal horns at 14 dpi of SNTR, SNTR+rAES, SNTR+CES and SNTR+iCES rats, with graphic representation of laminae I, II and III as regions of interest considered for quantification. Scale bar $100 \mu \mathrm{m}$. (B) Quantification of Iba1 immunoreactivity in the ipsilateral and contralateral dorsal horn of naïve and SNTR rats compared with the electrical stimulation treatments at 14 and $21 \mathrm{dpi}$. * $p<0.05$. 


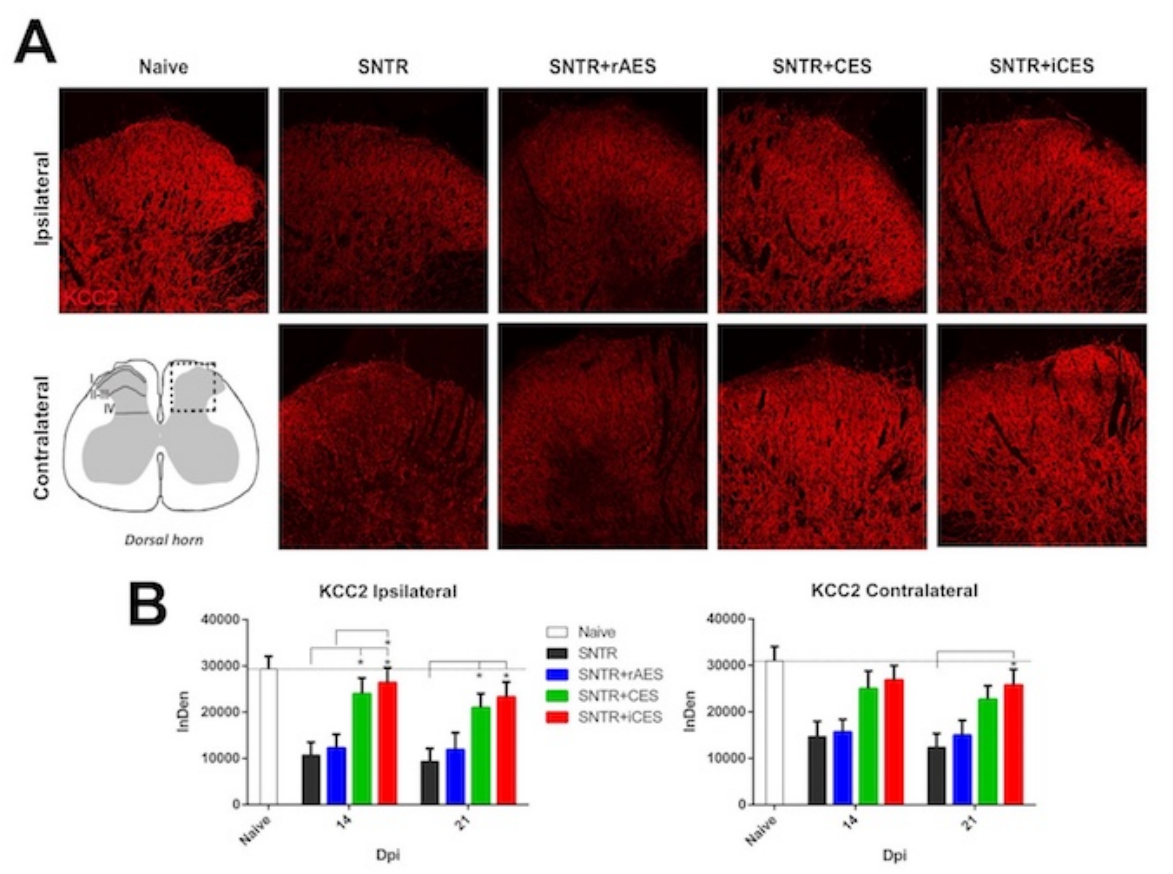

Figure 4. Chronic stimulation reverted the decrease of KCC2 expression in the dorsal horn after SNTR. (A) Representative confocal images of KCC2 immunoreactivity in the spinal dorsal horn of naïve rats, and in the ipsilateral and contralateral dorsal horns at $14 \mathrm{dpi}$ of SNTR, SNTR+rAES, SNTR+CES and SNTR+iCES rats, with graphic representation of laminae I, II and III as regions of interest considered for quantification. Scale bar $100 \mu \mathrm{m}$. (B) Quantification of KCC2 in the ipsilateral and contralateral dorsal horn of naïve, and SNTR rats compared with the electrical stimulation treatments at 14 and 21 dpi. $* p<0.05$.

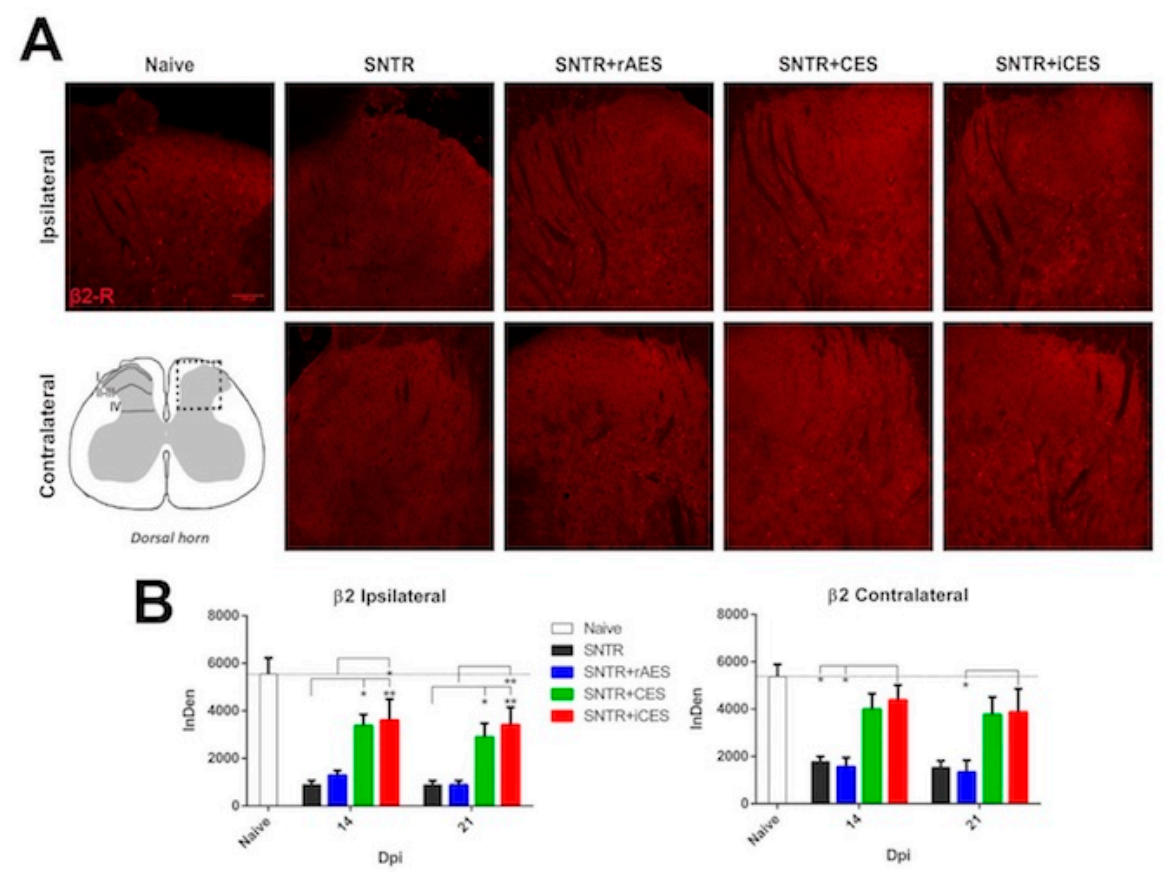

Figure 5. Chronic stimulation counteracted the decrease of $\beta_{2}$ receptor expression in dorsal horn after SNTR. (A) Representative confocal images of $\beta_{2}$ adrenergic receptor immunoreactivity in the spinal dorsal horn of naïve rats, and in the ipsilateral and contralateral dorsal horns at $14 \mathrm{dpi}$ of SNTR, SNTR+rAES, SNTR+CES and SNTR+iCES rats, with 
graphic representation of laminae I, II and III as regions of interest considered for quantification. Scale bar $100 \mu \mathrm{m}$. (B) Quantification of $\beta_{2}$ immunoreactivity in the ipsilateral and contralateral dorsal horn of naïve, and SNTR rats compared with the electrical stimulation treatments at 14 and 21 dpi. ${ }^{*} p<0.05, * * p<0.01$.
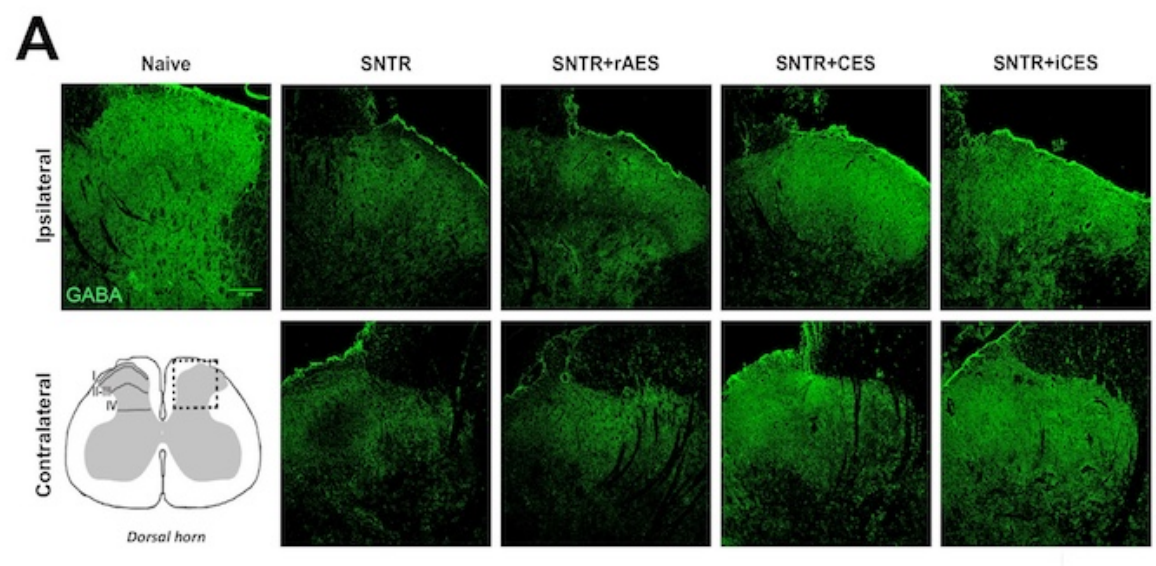

B
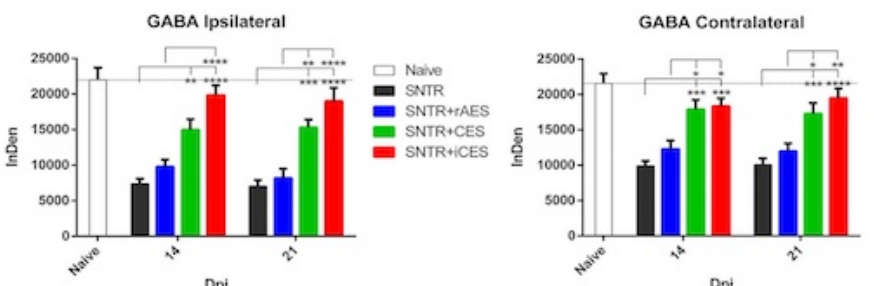

Figure 6. Chronic stimulation counteracted the decrease of GABA in dorsal horn after SNTR. (A) Representative confocal images of GABA immunoreactivity in the spinal dorsal horn of naïve rats, and in the ipsilateral and contralateral dorsal horns of SNTR, SNTR+rAES, SNTR+CES and SNTR+iCES rats at $14 \mathrm{dpi}$, with graphic representation of laminae I, II and III as regions of interest considered for quantification. Scale bar $100 \mu \mathrm{m}$. (B) Quantification of GABA in the ipsilateral and contralateral dorsal horn of naïve, and SNTR rats compared with the electrical stimulation treatments at 14 and $21 \mathrm{dpi}$. ${ }^{*} \mathrm{p}<0.05, * * \mathrm{p}<0.01, * * * \mathrm{p}<0.001$, $* * * * p<0.0001$. 